\title{
Effects of aerodynamic particle interaction in turbulent non-dilute particle-laden flow
}

\author{
Salewski, Mirko; Fuchs, Laszlo
}

Published in:

Journal of Turbulence

Link to article, DOI:

$10.1080 / 14685240802577879$

Publication date:

2008

Link back to DTU Orbit

Citation (APA):

Salewski, M., \& Fuchs, L. (2008). Effects of aerodynamic particle interaction in turbulent non-dilute particle-laden flow. Journal of Turbulence, 9(46), 1-23. https://doi.org/10.1080/14685240802577879

\section{General rights}

Copyright and moral rights for the publications made accessible in the public portal are retained by the authors and/or other copyright owners and it is a condition of accessing publications that users recognise and abide by the legal requirements associated with these rights.

- Users may download and print one copy of any publication from the public portal for the purpose of private study or research.

- You may not further distribute the material or use it for any profit-making activity or commercial gain

- You may freely distribute the URL identifying the publication in the public portal

If you believe that this document breaches copyright please contact us providing details, and we will remove access to the work immediately and investigate your claim 


\title{
Effects of Aerodynamic Particle Interaction in Turbulent Non-Dilute Particle-Laden Flow
}

\author{
Mirko Salewski $^{a, b}$ and Laszlo Fuchs ${ }^{b, c}$ \\ a) Ris $\varnothing$ National Laboratory for Sustainable Energy, \\ Technical University of Denmark, DK-4000 Roskilde, Denmark \\ b) Division of Fluid Mechanics, Department of Energy Sciences, \\ Lund University, SE-22100 Lund, Sweden \\ c) Linné Flow Centre, Department of Mechanics, \\ Royal Institute of Technology, SE-10044 Stockholm, Sweden
}

2008

\begin{abstract}
Aerodynamic four-way coupling models are necessary to handle twophase flows with a dispersed phase in regimes in which the particles are not dilute enough to neglect particle interaction but not dense enough to bring the mixture to equilibrium. We include an aerodynamic particle interaction model within the framework of large-eddy simulation (LES) together with Lagrangian particle tracking (LPT). The particle drag coefficients are corrected depending on relative positions of the particles accounting for the strongest drag correction per particle but disregarding many-particle interactions. The approach is applied to simulate monodisperse, rigid, and spherical particles injected into crossflow as an idealization of a spray jet in crossflow. A domain decomposition technique reduces the computational cost of the aerodynamic particle interaction model. It is shown that the average drag on such particles decreases by more than $40 \%$ in the dense particle region in the near-field of the jet due to the introduction of aerodynamic four-way coupling. The jet of monodisperse particles therefore penetrates further into the crossflow in this case. The strength of the counter-rotating vortex pair (CVP) and turbulence levels in the flow then decrease. The impact of the stochastic particle description on the four-way coupling model is shown to be relatively small. If particles are also allowed to break up according to a wave breakup model, the particles become polydisperse. An ad hoc model for handling polydisperse particles under such conditions is suggested. In this idealized atomizing mixture, the effect of aerodynamic four-way coupling reverses: The aerodynamic particle interaction results in a stronger CVP and enhances turbulence levels.
\end{abstract}




\section{Introduction}

The modeling of sprays in turbulent flows is still a challenge despite its importance in a number of industrial applications. These include fuel preparation for combustion, spray drying, or spray painting, to name just a few. An example flow topology of a fuel preparation system for gas turbines is the spray jet in crossflow (JICF) which we investigate here [1-5]. The spray is bent into the crossflow and in turn induces vortical structures such as the eminent counterrotating vortex pair (CVP). High aerodynamic strain on the droplets promotes fast breakup and subsequent vaporization of the droplets. The fuel gas can then mix with air prior to combustion so as to avoid the diffusion flame mode in which $\mathrm{NO}_{x}$ production is excessive [6]. The motion of sprays in turbulent flows is also a fluid mechanical problem of generic interest. In principle, flows with sprays are governed by the Navier-Stokes equations which are valid inside each droplet (the dispersed phase) and in the gas phase (the continuous carrier phase). Surface tracking techniques have been applied to solve for the motion, deformation, breakup, and coalescence of droplets $[7,8]$. However, the range of length scales inherent to typical sprays renders the surface tracking approach impossible for simulation of the motion of the entire spray. Hence one needs to introduce additional models to handle flows with sprays. Two common modeling approaches are the Euler/Euler and the Euler/Lagrange descriptions $[9,10]$. The carrier gas phase is usually described as a continuum in Eulerian framework. If the flow of the carrier gas phase is turbulent and has a large Reynolds number, it is usually impossible to resolve the small scale vortices by a Direct Numerical Simulation (DNS). Turbulence in the continuous phase is therefore commonly handled by Reynolds-Averaged Navier-Stokes (RANS) models or Large Eddy Simulation (LES). We apply the latter and discuss the application to two-phase flow in section 3 .

If one has a situation with very dense sprays, the droplets strongly interact with each other over short time scales and the dispersed phase can then be considered as a second continuum in equilibrium which has its own physical properties, e.g. viscosity or density (Euler/Euler). The equilibrium situation occurs for low Knudsen number of the dispersed phase $\left(K n_{d} \ll 1\right)$, defined as the mean free path of a droplet between two droplet-droplet interactions and the length scale over which average droplet properties vary. The interaction among the constituents of the two phases is accounted for through explicit interaction terms posing challenges for the modeling.

In the other extreme situation, the inter-droplet distance is much larger than the droplet size. For these dilute sprays, particles are tracked through the flow by integrating an equation of motion in Lagrangian coordinates. This approach has been dubbed Lagrangian Particle Tracking (LPT) or Euler/Lagrange. For very dilute sprays the effects of the droplets on the carrier phase are negligible. It is then acceptable to apply so-called one-way coupling between the two phases, i.e. momentum is transferred from the continuous phase to the dispersed phase but not vice versa. If momentum transfer from the droplets to the carrier phase is also accounted for, the phase coupling model is referred to as two-way 
coupling which will typically be necessary for less dilute sprays. For even less dilute sprays, there will be significant mutual interaction not only between the phases but also among the droplets themselves. Such droplet interaction models have been named four-way coupling [11] inspired by the terms two-way coupling for dilute sprays and one-way coupling for very dilute sprays. Droplet interaction can be categorized as direct (collision) or indirect (aerodynamic). Droplet collision models are widely available, but aerodynamic four-way coupling has often been disregarded. Collisions have stronger effects than aerodynamic interactions in most circumstances, but there are parameter regimes in which this may not be so, for example when collisions are rare but the particle density is high. An example is the airblast atomization (as opposed to pressure atomization) for which the droplets accelerate strongly and the average droplet spacing increases.

In many cases of engineering interest, the spray is not as dense as to allow an equilibrium in the interaction among the droplets but not as dilute as to neglect the aerodynamic interaction between them, either. A model premise of LPT is that droplets are isolated, i.e. there are supposed to be no other droplets in the flow when the drag is computed as discussed further in section 2. However, particles may cross each other's wakes and displace fluid and thereby remotely interact with each other. If the particles cannot be assumed to be isolated, application of LPT to such regimes is inconsistent [12]. In this respect, there is a gap in the applicability between Euler/Euler and Euler/Lagrange models for multiphase flows which are not dilute but not dense enough, either. A model for aerodynamic interaction of particles, as we propose here, can extend the range of validity of LPT to somewhat denser spray situations. In the following, we consider an idealized flow with rigid, spherical particles and ignore deformation, atomization, collision, or internal circulation. Hence we refer to the dispersed phase as "particles" from now on. Though particle interaction between a pair of, a triplet of, or a few particles in the microscopic picture has been studied by many groups, the application of these particle interaction studies to LPT in the macroscopic picture has not often been addressed. The terms "microscopic" and "macroscopic" refer to our particular application and make sense only in this respect as the Navier-Stokes equations are of course Reynolds number invariant.

Investigations of aerodynamic particle interaction in the microscopic picture are numerous. For a pair of spheres in various configurations, significant aerodynamic interactions were demonstrated [13-21]. For example, the aligned tandem formation results in drag reductions of $30 \%$ on the particle in the wake for an inter-particle spacing $L / D_{p} \sim 6$ [22]. Also chains of particles [23] and three spheres in a straight line at various angles [18] and in L-formation and side-byside-by-side formation [24] have been found to show remote interaction effects. Flow around a pair of spheres of unequal size has also been computed [20].

In the macroscopic picture, Silverman and Sirignano [25] developed particle interaction models to correct the drag coefficient in an LPT calculation. Their drag correction factor was, however, based on assumed stochastic positions of the interacting particles. Aerodynamic particle interaction has also been studied extensively in the context of atmospheric clouds by LPT together 
with DNS for turbulence in the background air [26-28]. In these simulations, the suspended particles are much smaller than the Kolmogorov scales and have particle Reynolds numbers below one. A scale separation argument then allows a decoupled solution of a Stokes problem for the particles and DNS for the background. The volume fraction of the particles in the cloud was on the order of $10^{-6}$. Even at these low densities, significant aerodynamic interaction was found. We investigate here a much denser spray with volume fractions on the order of $10^{-2}$. In the spray JICF application, particle sizes on the order of the Taylor micro-scale are not uncommon which poses challenges for the modeling.

We solve the macroscopic particle-laden flow by LES/LPT including a parametrization of microscopic flow solutions surrounding pairs of equally sized spheres as has been computed for various Reynolds numbers, orientations and interaction distances (see figure 1) [22]. The particle equation of motion is modified to include a precomputed drag correction factor which depends on theses parameters. The model handles aerodynamic interaction of monodisperse particles, but an extension to include polydisperse particles is suggested. The model is presented in section 2. Section 3 describes the LES of the turbulent motion in the continuous phase. In section 4 , the boundary conditions are defined. The proposed particle interaction model is applied in LES/LPT of dense monodisperse and polydisperse particle jets in crossflow in section 5 . In the section 5.1, we consider the effects of four-way interaction as compared to the two-way interaction model. Each particle is tracked individually, i.e. a computational particle represents one physical particle. The effect of stochastic particle treatment [29] and polydisperse spray with a breakup model [30] are addressed in sections 5.2 and 5.3, respectively. The four-way coupling substantially reduces the drag on the particles in the near-field of the jet, and thus the particles follow higher trajectories. Additionally, the four-way coupling reduces turbulence production. However, if the particles break up (i.e. they are droplets), four-way coupling enhances turbulence.

\section{Lagrangian Particle Tracking}

We briefly review underlying assumptions of LPT. If it is assumed that the particles are spherical, rigid, and isolated, and that the particle Reynolds number vanishes, one arrives at the so-called BBO equation (Basset-Boussinesq-Oseen) which was derived by Maxey and Riley [31]. This equation does not include Saffman- or Magnus lift forces or Mach number effects. The BBO equation is also valid in turbulent flow if the particles are much smaller than the Kolmogorov scales as for example in atmospheric clouds [26-28]. If this condition is not met, as in many engineering applications, then the particles also interact with turbulence, leading to turbulence production or dissipation [9]. For high particle Reynolds number, one replaces the Stokes drag by an empirical relation for steady-state aerodynamic drag of an isolated particle. If the density ratio of the dispersed phase $\rho_{d}$ and the continuous phase $\rho_{c}$ is large $\left(\rho_{d} / \rho_{c} \gg 1\right)$ one may neglect several terms in the BBO equation, for example the Basset force, 
the virtual mass force, and the force due to flow acceleration. If the Froude number is large, one may also neglect gravity. The Froude number is defined as $F r=\frac{|\vec{v}-\vec{u}|}{\sqrt{g D_{p}}}$, where $\vec{v}$ is the particle velocity, $\vec{u}$ is the local continuous phase velocity at the particle location, $D_{p}$ is the diameter of the particle, and $g$ is the gravitational acceleration. It is also frequently assumed that the particles occupy no volume in the continuous phase.

The motion of the particles in flows of engineering relevance is then often tracked by the Lagrangian approach summarized in equations (1) to (4). One obtains the particle acceleration from Newton's law and the velocities and positions by integration.

$$
\begin{aligned}
& \frac{d \vec{v}}{d t}=-\frac{3}{4} \frac{\rho_{c}}{\rho_{d}} \frac{1}{D_{p}} C_{D}|\vec{v}-\vec{u}|(\vec{v}-\vec{u}) \\
& \frac{d \vec{x}}{d t}=\vec{v}
\end{aligned}
$$

The drag coefficient $C_{D}$ in equation (1) is a function of the particle Reynolds number $R e_{p}$ as given by equation (3). The particle Reynolds number is defined in equation (4) where $\nu_{c}$ is the kinematic viscosity of the continuous phase. Equation (3) is valid for a single, isolated sphere in a uniform flow among other assumptions and may be regarded as empirical.

$$
\begin{aligned}
C_{D} & = \begin{cases}\frac{24}{R e_{p}}\left(1+\frac{1}{6} R e_{p}^{2 / 3}\right) & \text { for } R e_{p} \leq 1000 \\
0.424 & \text { for } R e_{p} \geq 1000\end{cases} \\
R e_{p} & =\frac{|\vec{v}-\vec{u}| D_{p}}{\nu_{c}}
\end{aligned}
$$

\subsection{LPT with Aerodynamic Four-Way Coupling}

The aerodynamic four-way coupling is modeled by a precomputed drag correction factor $f$, and the right hand side in equation (1) is adjusted by this factor. The correction factor $f$ has been computed previously depending on the interaction distance $r_{i n t}$, the angle $\alpha$, and the particle Reynolds number $R e_{p}[22]$. We assume here that the flow conditions for both particles are similar. Also, it is supposed that the dynamics of the particle motion is negligible, i.e. the applied drag correction factors are results of steady-state flow computations. The parametrization is summed up in look-up tables provided in the appendix (the parameters therein are defined in section 2.2). $C_{D}$ given by equation (3) which is valid for a rigid, isolated sphere. The final drag is changed according to equation (5) when a second particle is close enough. The equation of particle motion, with a correction for aerodynamic four-way coupling, reads:

$$
\frac{d \vec{v}}{d t}=-\frac{3}{4} \frac{\rho_{c}}{\rho_{d}} \frac{1}{D_{p}} C_{D} f|\vec{v}-\vec{u}|(\vec{v}-\vec{u})
$$

It may be noted that shear effects could be modeled in an analogous way. Lift coefficients due to presence of other particles in the microscopic picture have 


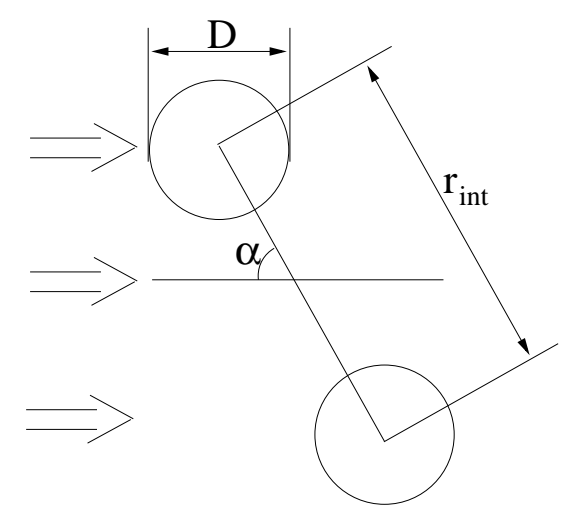

Figure 1: Orientation and length scales given by a pair of particles in a flow

\section{missed interaction}

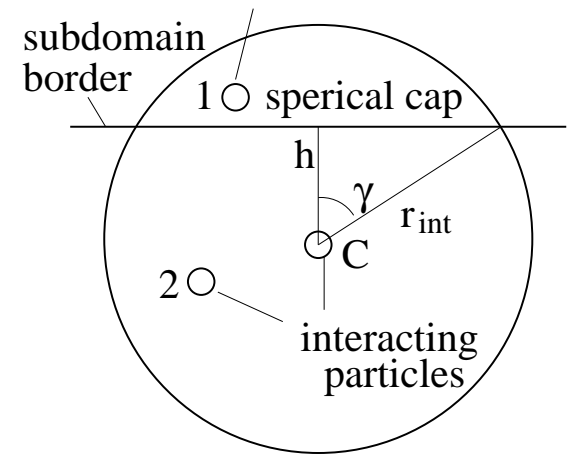

Figure 2: Particles close to the boundary of a subdomain

been computed [22]. This has not been included in the present modeling and is a topic of future investigations.

\subsection{Conditions for Modeled Particle Coupling}

If the distance between two particles is not large or when the effects of the wake of another particle have not dissipated, the interaction between these particles may be large. The aerodynamic interaction is a remote interaction (as opposed to the direct contact as in collision models) and hence one must introduce the notion of the particle interaction distance $r_{i n t}$. The interaction distance is the distance between the centers of two particles as sketched in figure 1. The length scale of typical interaction distances can be compared to turbulence length scales: Since the Stokes number based on integral scale is $S t_{0} \sim 0.1-1$, the ratio of particle size $D_{p}$ and the integral scales is of order $R e_{T}^{-1 / 2}$. Thus, the particle diameter and therewith the interaction distance are on the order of the Taylor micro-scale. The integral scale Stokes number $S t_{0}$ and the turbulence Reynolds number $R_{T}$ are defined in section 3.3, respectively. A second quantity defined in figure 1 is the angle $\alpha$ between the wind direction and the line between the particle centers.

All interactions at a distance of more than six particle diameters are neglected as stated in equation (6). This is justified since the drag correction factor $f$ approaches unity for large $r_{\text {int }}$, though it can still be small in the wake 
even at larger distances (meaning strong particle interaction). Secondly, if the particle velocity vectors span an angle larger than $5^{\circ}$, then the particle trajectories diverge fast, resulting in a small particle interaction time, and it is assumed that the interaction can be neglected as summarized in equation (7). The particular values on the right hand sides of equations (6) and (7) are chosen such that only the strongest interactions are accounted for. The strength of fourway coupling of course decreases for larger interaction distances $r_{\text {int }}$ and angles between the velocity vectors. For example, one could have allowed interactions up to ten particle diameters or up to an angle of $10^{\circ}$. This would result in a further increase of the effect of aerodynamic four-way coupling, but at a larger computational cost.

$$
\begin{aligned}
\frac{r_{i n t}}{D_{p}} & <6 \\
\frac{\left|\vec{v}_{1} \cdot \vec{v}_{2}\right|}{\left|\vec{v}_{1}\right|\left|\vec{v}_{2}\right|} & <\cos 5^{\circ}
\end{aligned}
$$

The particle cloud may be so dense that a given particle may interact with several other particles (i.e. satisfy the above mentioned conditions with several partners). As the present look-up tables are valid for interaction among a pair of particles, only one interaction partner per particle is allowed. This limitation can be remedied by creating a data base for particle configurations that contain more particles as e.g. in references [18] or [24]. This would require a further parametrization and there is a limit where the efficiency of the approach would be hampered. Thus, the approach so far has been limited to static interaction between two particles. The strongest interaction is chosen, and the other interactions are ignored.

Note that these conditions imply that the inconsistency of the LPT is remedied only if the particle loading is not too high, leading to multiple-particle interactions. The assumption of isolated pairs of particles (if the above conditions are met) is, however, a better approximation in a non-dilute spray than the assumption of isolated particles.

\subsection{Domain Decomposition for Four-Way Coupling}

Four-way coupling requires substantial amount of computer time since the computational effort for particle interaction models scales with the square of the particle number whereas computational effort for particle transport, evaporation, or breakup models are linearly proportional to the particle number. This fact limits the number of particles that can be used in four-way coupling models and has led to the proposition of creating fictitious interaction partners in collision models which also results in linear scaling [32]. We speed up the computation by a domain decomposition technique in which the $N$ particles are then only allowed to interact with other particles within the same subdomain $S$. Suppose that the subdomains are chosen such that each subdomain contains an equal number of particles, $\frac{N}{S}$. The number of interactions then scales with 
$S\left(\frac{N}{S}\right)^{2} \sim \frac{N^{2}}{S}$, and the computational time thus scales inversely with the number of subdomains. However, the disadvantage of this domain decomposition is that not all possible interactions are discovered in this way as is illustrated in figure 2: Several particles close to a border between two subdomains are sketched. The circle represents the maximum interaction distance of the center particle, and hence particle 1 and particle 2 can interact with the center particle since they satisfy the criterion given by Equation (6), i.e. they are close enough to the center particle. However, particle 1 is outside the subdomain, and this potential interaction is therefore overlooked due to the domain decomposition. We estimate in the following the resulting error for cubic subdomains with a side length of $100 D_{p}$.

Particles will not miss any interactions if they are further away from the boundary of the subdomain than the interaction distance, e.g. for an interaction distance of $r_{i n t}=2 D_{p}$, no interactions can be missed for particles in a cube with a side length of $96 D_{p}$ centered within the subdomain with a side length of $100 D_{p}$. Therefore, $\frac{96^{3}}{100^{3}}=88 \%$ of the interactions at $r_{\text {int }}=2 D_{p}$ are surely found because the particles are far from the subdomain boundaries. This percentage depends, however, on the interaction distance: $94 \%$ of the particles are so distant from the boundary of the subdomain that they could not possibly miss interactions at an interaction distance of $r_{i n t}=1 D_{p}, 88 \%$ for $r_{i n t}=2 D_{p}$ (as explained above), $83 \%$ for $r_{i n t}=3 D_{p}, 79 \%$ for $r_{i n t}=4 D_{p}, 73 \%$ for $r_{i n t}=5 D_{p}$, and $68 \%$ for $r_{\text {int }}=6 D_{p}$. If the particles are close to the boundary, still not all interactions will be missed: Even a particle located on the border between two subdomains only misses $50 \%$ of all possible interaction partners on average. The situation is illustrated in figure 2. The particles can interact with partners within the sphere of radius $r_{i n t}$. Suppose that the particle is situated at a distance $h$ from the border of the subdomain. Then the particle misses interactions with other particles within the spherical cap outside the subdomain. The volume of such a spherical cap divided by the volume of a full sphere is dependent on the angle $\gamma$ with $\cos \gamma=h / r_{i n t}$. Thus, one can estimate how many interactions are missed for any given $\gamma$ :

$$
\frac{V_{\text {cap }}}{V_{\text {sphere }}}=\frac{1}{4}\left(2-2 \cos \gamma-\cos \gamma \sin ^{2} \gamma\right)
$$

As the position of the border between the subdomains is arbitrary, one can simply average over all positions. Thus, the average volume of the spherical cap (which contains the particles for which interaction are missed) divided by the total volume of the sphere is:

$$
\begin{aligned}
\frac{\overline{V_{\text {cap }}}}{V_{\text {sphere }}} & =\frac{\int_{0}^{\frac{\pi}{2}} \frac{V_{\text {cap }}}{V_{\text {sphere }}} d \hat{\gamma}}{\frac{\pi}{2}}=\frac{1}{2 \pi} \int_{0}^{\frac{\pi}{2}}\left(2-2 \cos \hat{\gamma}-\cos \hat{\gamma} \sin ^{2} \hat{\gamma}\right) d \hat{\gamma} \\
& =\left.\frac{1}{2 \pi}\left(2 \gamma-2 \sin \gamma-\frac{1}{3} \sin ^{3} \gamma\right)\right|_{0} ^{\frac{\pi}{2}}=\frac{1}{2 \pi}\left(\pi-\frac{7}{3}\right) \approx 0.13
\end{aligned}
$$

Following the arguments above, one can estimate that even for particles close to the border only about $13 \%$ of the situations that should use four-way 
interactions are neglected (disregarding the edges and corners of the cube where particles are close to three and seven other subdomains, respectively). Hence the estimates for missed interactions are as follows: $0.8 \%$ for $r_{\text {int }}=1 D, 1.5 \%$ for $r_{i n t}=2 D, 2.2 \%$ for $r_{i n t}=3 D, 2.8 \%$ for $r_{i n t}=4 D, 3.5 \%$ for $r_{i n t}=5 D$, and $4.1 \%$ for $r_{i n t}=6 D$. Note that the interactions over a distance of $1 D$ are the strongest and therefore the most important to find. It can be concluded that only an insignificant number of interactions are disregarded if the subdomain size is about 100 particle diameters.

\section{Large Eddy Simulation}

\subsection{Governing Equations}

A volume averaging procedure ensures that macroscopic gas properties are defined continuously at every point in the domain, even though the dispersed phase obviously also occupies some space. The continuous phase is treated as incompressible flow (low Mach number assumption) and as the Mach number of the present flow is $M a=0.25$, compressibility effects are of order $M a^{2} \approx$ $6 \%$. The presence of the dispersed phase may be taken into account through source terms in the averaged equations. The volume fraction of the dispersed phase is about 0.06 up to 6 nozzle diameters but decreases along the trajectory of the particles, such that it has dropped to under 0.01 except within about 30 nozzle diameters [12]. Therefore, the volume fraction of the continuous phase is set to unity. The non-dimensional continuity and momentum equations are filtered to separate the large scale structures from the small scale structures. The filtered equations for continuity and momentum are presented in equation (11) and equation (12), respectively.

$$
\begin{gathered}
\frac{\partial \widetilde{u}_{i}}{\partial x_{i}}=0 \\
\frac{\partial \widetilde{u}_{i}}{\partial t}+\widetilde{u}_{j} \frac{\partial \widetilde{u}_{i}}{\partial x_{j}}=-\frac{\partial \widetilde{p}}{\partial x_{i}}+\frac{1}{R e} \frac{\partial^{2} \widetilde{u}_{i}}{\partial x_{j}^{2}}+\widetilde{\dot{M}}_{s, i}-\frac{\partial}{\partial x_{j}}\left(\widetilde{u_{i} u_{j}}-\widetilde{u}_{i} \widetilde{u}_{j}\right)
\end{gathered}
$$

$\widetilde{u}_{i}$ are the filtered velocities and $\widetilde{p}$ is the filtered pressure. Re is the Reynolds number of the gas phase based on a characteristic length scale $l_{0}$ and a velocity scale $U$ of the flow. $\frac{\partial}{\partial x_{j}}\left(\widetilde{u_{i} u_{j}}-\widetilde{u}_{i} \widetilde{u}_{j}\right)$ are the so-called subgrid scale (SGS) stresses discussed in the next section. $\widetilde{\dot{M}}_{s, i}$ are the filtered source terms for the momentum equations which account for the momentum transfer from the dispersed phase to the continuous phase. The source terms are computed from the rates of momentum change in the dispersed phase, depending on the rate of change of particle momentum $m_{p} v_{p}$ and the number of particles in a parcel $N_{p}$ (which is set to unity in section 5.1 but varies in sections 5.2 and 5.3 ).

$$
\widetilde{\dot{M}}_{s, i}=-\frac{L}{\rho_{c} U^{2}} \int N_{p} \frac{d{\widetilde{m_{p} v}}_{p, i}}{d t} d v d r
$$


The change of particle momentum can be computed since the particle mass and the particle velocity are readily available for each particle (or parcel) in the Lagrangian approach. One obtains point sources of momentum which are subgrid terms. They are therefore handled consistently in LES by the filtering process.

\subsection{Subgrid-Scale Modeling}

Small eddies are universal for all flows with sufficiently high Reynolds number and therefore amenable to generally valid models, the Subgrid-Scale (SGS) models. In this work, the MILES (Monotonically Integrated Large Eddy Simulation) approach is employed [33], also sometimes called implicit turbulence model, meaning formally that the SGS stresses are set to zero. This is justifiable if the unresolved eddies contain little kinetic energy as compared to the resolved eddies, and the unresolved eddies can therefore be neglected. This approach has an increasing acceptance as due to increase in computational resources a larger part of the turbulence spectrum can be resolved and the unresolved kinetic energy therefore declines with increasing resolution. In this respect, LES is an approximation to DNS, and therefore it is conceptually acceptable that no explicit SGS model is required [34-36]. SGS models should drain energy at the smallest resolved waves but should be negligible for the largest waves. Stable high-order numerical schemes such as the employed Weighted Essentially Non-Oscillatory (WENO) scheme [37] can be used for this dissipation of kinetic energy from the smallest resolved scales, and this leads to the idea to use no explicit turbulence model. It has also been demonstrated that the discretization error of numerical schemes is on the same order as the computed SGS flux in a wide range of resolvable waves $[38,39]$. Numerical schemes therefore intrinsically interfere with subgrid models.

\subsection{LES with LPT}

LES is a versatile alternative for multiphase flows with a dispersed phase since results of Wang and Squires [40] and Yeh and Lei [41] suggest that particle dispersion depends mainly on the large scale motion. This can also be inferred from an argument based on the Stokes number. The Stokes number is the ratio of particle momentum relaxation time $\tau_{v}$ and a typical flow time scale $\tau_{f}$ :

$$
S t=\frac{\tau_{v}}{\tau_{f}}
$$

If $S t>>1$, the particle motion is mainly ballistic, whereas if $S t<<1$, the particle motion closely follows the fluid motion. The particle momentum relaxation time is often based on Stokes flow past a sphere:

$$
\tau_{v}=\frac{\rho_{d}}{\rho_{c}} \frac{D_{p}^{2}}{18 \nu_{c}}
$$


A typical flow time scale is the integral time scale which may be estimated as:

$$
\tau_{0}=\frac{l_{0}}{u_{0}}
$$

In a turbulent flow, however, there is a wide range of time scales $\tau_{f}$ : The large scale motion has longer time scales than small scale motion, and thus a Stokes number based on large time scales $\tau_{0}$ is considerably smaller than a Stokes number based on small time scales $\tau_{k}$. Limiting Stokes numbers are based on the integral scale $S t_{0}=\tau_{v} / \tau_{0}$ for large flow time scales and on the Kolmogorov time scales $S t_{k}=\tau_{v} / \tau_{k}$. Since $\tau_{0} / \tau_{k} \sim R e_{T}^{1 / 2}$ where $R e_{T}$ is the turbulence Reynolds number based on the integral scales $u_{0}, l_{0}$, and $\nu_{c}$ (equation (18)), one can determine a range for the Stokes number between $S t_{k}$ and $S t_{0}$ which are related by:

$$
\begin{aligned}
S t_{k} & \sim R e_{T}^{\frac{1}{2}} S t_{0} \\
R e_{T} & =\frac{u_{0} l_{0}}{\nu_{c}}
\end{aligned}
$$

Equation (17) indicates that particle dispersion mainly depends on large scale vortices compared to small scale vortices to leading order, if the turbulent Reynolds number is large, as has also been observed [40,41]. This argument makes LES a sensible turbulence model since the large scale eddies, with which the particles mainly interact, are resolved. In RANS turbulence models, also the large scales of the turbulence spectrum are modeled, and therefore also the leading effects on particle dispersion. One may note that LES together with LPT is not self-evident. If the Stokes number $S t_{0}$ is not small (i.e. of order unity) then the ratio of the particle size to the integral length scale is of order $R e_{T}^{-1 / 2}$, which is the same order as the Taylor micro-scale. For a reasonably resolved LES, the grid size is of the same order. Thus, the assumption that the particles are so small that they are assumed not to displace the carrier fluid, is inconsistent with the LES-LPT approach if the particles are not so small that they follow the carrier fluid.

\subsection{Numerical Methods}

The equations of motion are discretized on a staggered grid with improved nearwall resolution due to grid stretching as described in section 4 . The convective terms are discretized with a fifth-order WENO scheme as mentioned before [37] and the diffusive terms with a fourth-order central scheme. The equations are integrated in time explicitly with a third-order Runge-Kutta scheme. Pressure corrections are obtained from a Poisson equation which is relaxed with a pointwise Gauss-Seidel iteration scheme. The convergence is accelerated by the multigrid method. The flow solver is described in more detail in reference [34]. 


\section{Case Description}

The baseline case is a spray jet in crossflow (JICF) which has been investigated using phase-Doppler anemometry (PDA), Mie-scattering, and shadowgraphy $[42,43]$. Spray was injected into a pressurized channel $(5.8 b a r)$ with crossflow. The nozzle diameter is $0.45 \mathrm{~mm}$, the channel height is $40 \mathrm{~mm}$, the gas velocity $U=100 \mathrm{~m} / \mathrm{s}$. The momentum flux ratio, defined by equation (19), is $J=18$ for the baseline case $[42,43]$.

$$
J=\frac{\left(\rho U^{2}\right)_{\text {jet }}}{\left(\rho U^{2}\right)_{\text {crossflow }}}
$$

The computational channel consists of two channels, each with dimensions of $(x, y, z)=(90,90,270)$ nozzle diameters (figure 3$)$. Both channels have no-slip conditions at the top and bottom wall and have periodic conditions in lateral direction. The first channel has also periodic conditions in streamwise direction, and simulates therefore simply a turbulent channel flow. The purpose of this first channel is to compute the inflow boundary conditions for a second channel, in which the transverse jet is injected. The outflow boundary condition of the second channel is a flux conserving zero-gradient. The center of the nozzle is defined to be the origin of the coordinate system in the following figures. The particles are assumed to bounce elastically at the walls; the lateral direction is assumed to be periodic. Particles are destroyed at the outlet of the flow domain. The flow Reynolds number is set to $R e=10000$ in the computation though the experiment had a higher Reynolds number. Here, no attempt to simulate the experimental conditions by LES is undertaken concordant with the neglect of other models simulating droplet behavior, e.g. atomization. The computational grid is structured with staggering of the velocity and pressure nodes. The grid has about 3.8 million cells, with 80 cells in $\mathrm{x}$ and y directions (across the channel) and 480 cells in $\mathrm{z}$ direction (along the channel). This cell size in $\mathrm{x}$ and $\mathrm{z}$ directions is on the order of the nozzle diameter. The grid is stretched in y direction to increase the near-wall resolution $\left(y^{+} \sim 3\right)$.

\section{$5 \quad$ Results}

The given baseline case has been studied in several publications in which gas velocities and particle volume fluxes are presented [42-44]. Figure 4 shows a comparison of a computed volume fraction profile to the corresponding PDA measurement provided by Rachner et al. [43]. The line and the circles represent computed and measured results, respectively. In this comparison, droplet breakup and collision have been modeled. A $\chi^{2}$-distribution was used with the mean droplet diameter set to equal the nozzle size (blob injection). The initial velocity of the particles is given by the momentum flux ratio. The profiles are taken along a horizontal line 18 nozzle diameters above the wall from which the spray is ejected and 178 nozzle diameters downstream of the nozzle. The simulation shows less dispersion than the PDA measurement. There could be several 


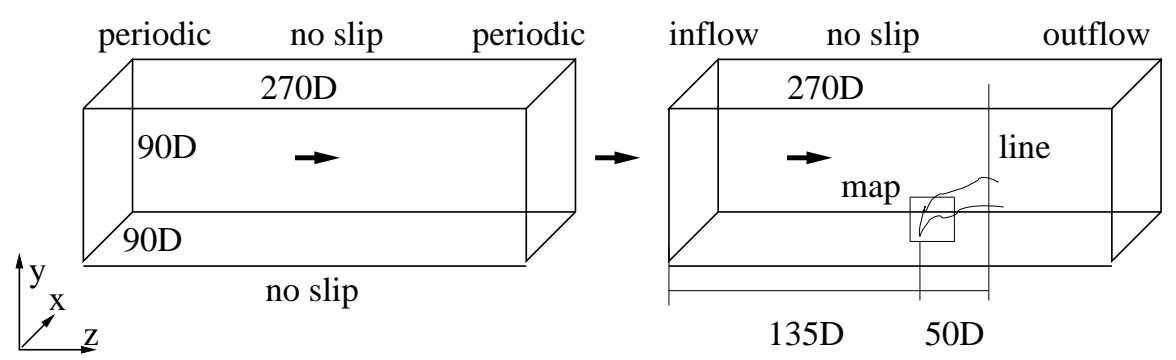

Figure 3: Geometry of the spray jet in crossflow

reasons for this discrepancy. For the LES, we have assumed an infinitely long channel, and this is of course not the real geometry in the experiment. This may lead to different turbulence levels and spectral content of the turbulence which is a highly important source of dispersion. Secondly, PDA measurements have usually a high uncertainty due to the assumed spherical shape of the droplets. On may also note that the experimental data is not symmetric about $x / D=0$ whereas the simulation is symmetric. This suggests asymmetric secondary flow structures in the experiments which will not occur in the simulation with the assumed boundary conditions. The numerical accuracy is analyzed in [45]. For example, the trajectory height is within $5 \%$ for grids from 1.4 to 3.8 million cells. In all, we find the agreement of the simulation and the experiment satisfactory. More details about the modeling of this polydisperse spray and the sensitivity to the particle diameter, the momentum flux ratio, and spray submodels for evaporation, breakup, and collision is further addressed in references [12,45-47].

\subsection{Direct Particle Treatment}

In this section, the effects of four-way coupling on the flow are presented for cases in which individual particles have been tracked. Each computational particle represents exactly one physical particle. As mentioned before, we consider initially the particles as being solid, rigid, and equally sized spheres with no break-up, collision, deformation, or evaporation. This idealized situation does not resemble a realistic spray, but is intended to elucidate the effects of four-way coupling more clearly without masking due to other models such as atomization or droplet collision. In the following, the momentum flux ratio is increased to $J=43$ to study an even more dense spray.

Figure 5 shows the average drag correction factors $f$ in the near-field of the liquid jet for particle diameters of $D_{p}=20 \mu \mathrm{m}$ and $D_{p}=40 \mu \mathrm{m}$. The position of the observation window in figure 5 is marked as "map" in figure 3 . The difference in particle size constitutes a substantial difference when one has a spray in mind 


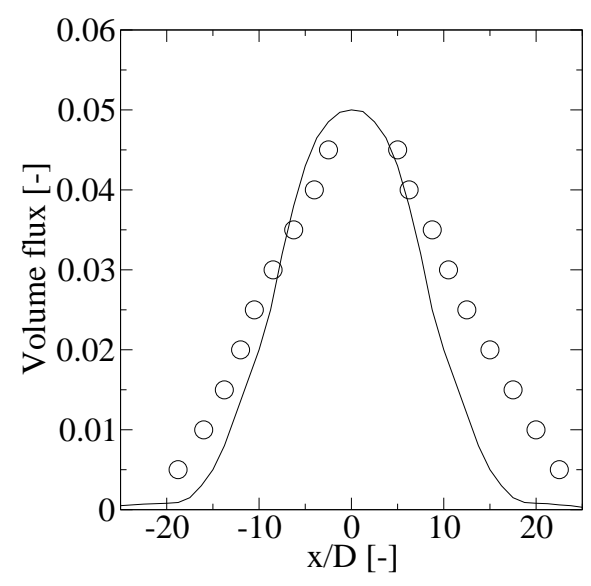

Figure 4: Comparison of the computation to experimental data $[42,43]$
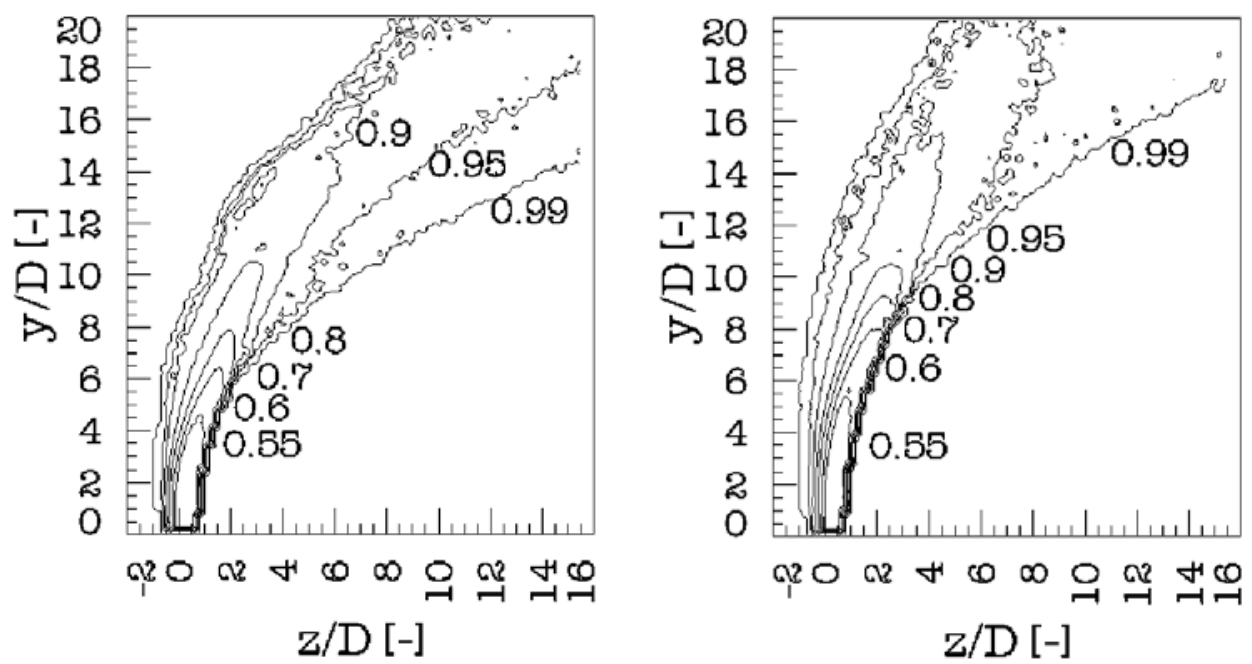
a) $D_{p}=20 \mu m$
b) $D_{p}=40 \mu m$

Figure 5: Average drag correction factors $f$ for non-stochastic particle treatment 


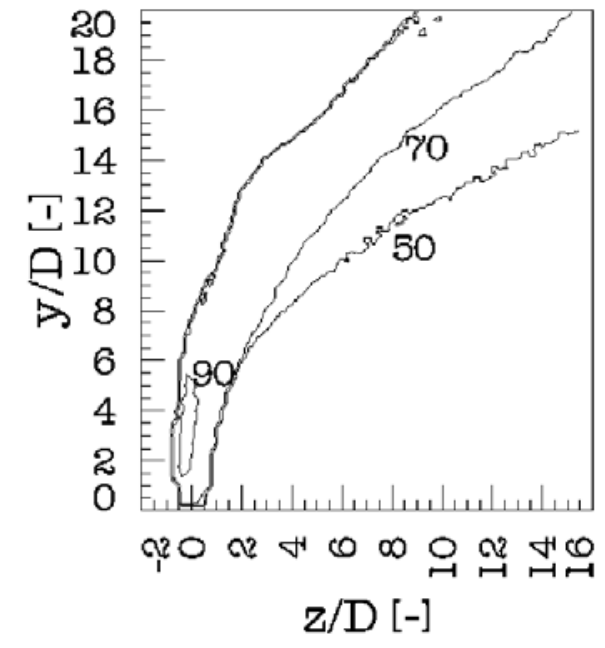

a) $D_{p}=20 \mu m$

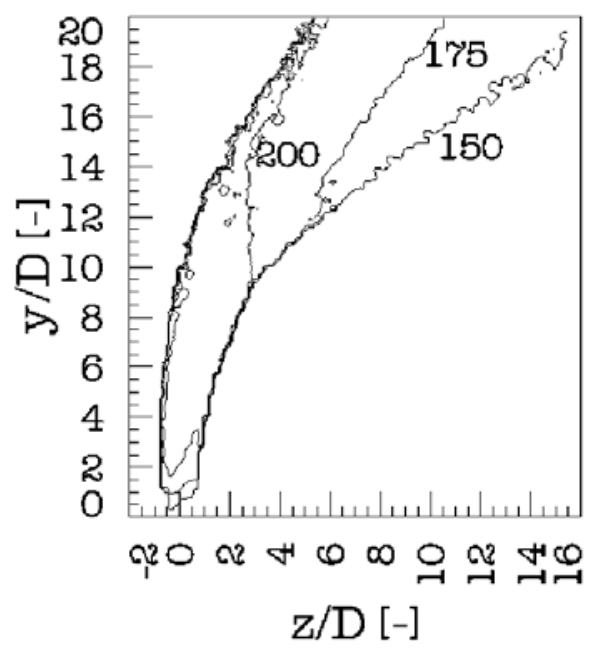

b) $D_{p}=40 \mu m$

Figure 6: Average particle Reynolds numbers $R e_{p}$

since the Stokes number is proportional to the square of the particle size. The Stokes number, based on the integral flow time scale and the particle momentum relaxation time, of these particles is on the order of $S t_{0} \sim 0.1-1$ (of course the Stokes number is larger by a factor four for the larger particles). Secondly, this difference is manageable with the available computational resources, keeping in mind that the particle number increases with the inverse cube of the diameter in direct particle treatment. The number of particles is thus eight times larger in the case with $D_{p}=20 \mu \mathrm{m}$. The volume fraction $\alpha$ of the dispersed phase is identical for both particle diameters, and hence the particle spacings $\frac{D_{p}}{L}$ are also identical as shown by equation (20) [9].

$$
\alpha=\frac{\pi D_{p}^{3}}{6 L^{3}}
$$

The drag on the particles decreases by as much as $40 \%$ within six nozzle diameters from the nozzle and by at least $10 \%$ within 16 nozzle diameters. These low drag correction factors (meaning strong aerodynamic particle interaction) occur for particles which follow other particles in their wake, analog to the considerable drag reductions a cyclist or swimmer can experience in the wake of fellow sportsmen. The drag correction factors are similar for both particle diameters.

As the drag coefficient and the drag correction factor are functions of the 


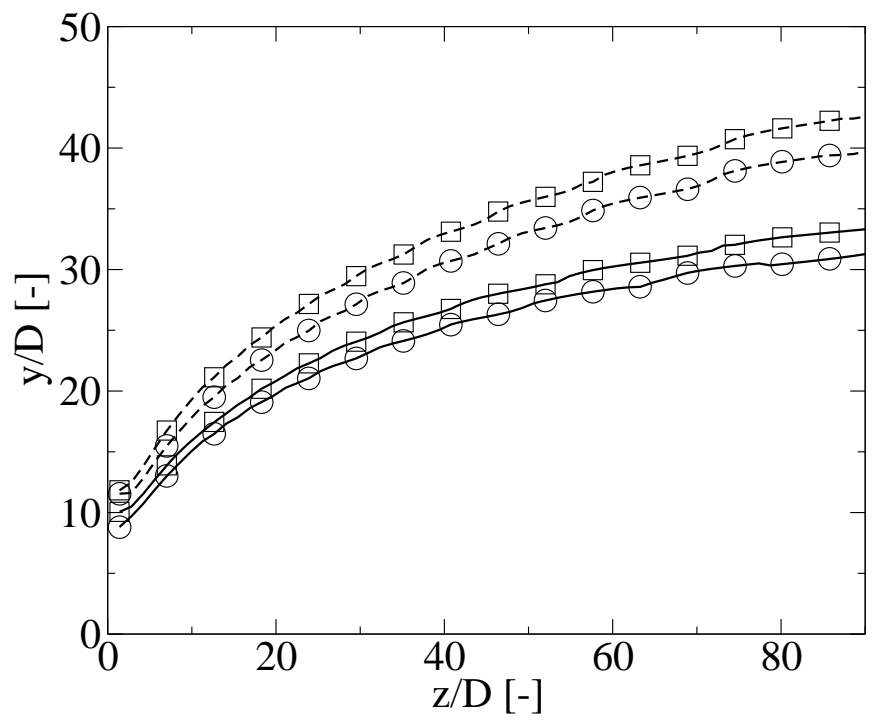

Figure 7: Mean particle trajectory, $-D_{p}=20 \mu m,---D_{p}=40 \mu m, \bigcirc$ two-way coupling, $\square$ four-way coupling

particle Reynolds number $R e_{p}$, defined in equation (4), maps for average particle Reynolds numbers are plotted in figure 6 . The position of the window is the same as for the drag coefficient and marked in figure 3. One may note the wide range of particle Reynolds numbers that characterizes the macroscopic flow. The microscopic flow around each particle changes significantly with the particle Reynolds number [22], and figure 6 indicates the relevant parameter regime. The particle Reynolds numbers are smaller for the small particles (figure 6a) since firstly the length scale is half as large as for the large particles (figure 6b) and secondly the slip velocities are smaller since the particle Stokes numbers are lower. The peak particle Reynolds numbers for small particles are below 100 whereas peak values for large particles are beyond 200 .

Figure 7 demonstrates that four-way coupling increases the particle penetration. The lines represent the mean particle trajectory, defined as the locus of maximum particle volume fraction in the centerplane. The trajectories are about $7 \%$ higher if aerodynamic four-way coupling is allowed for, both for small and large particles. The reason for the higher trajectories is the reduction in drag coefficient due to four-way coupling: Since the drag coefficient is lower, the particles do not decelerate as fast in $y$-direction and do not accelerate as fast in $z$-direction, leading to higher trajectories. Large particles penetrate further into the crossflow than small particles as aerodynamic forces scale with the square of the particle diameter whereas inertial forces scale with the particle volume. The ratio of aerodynamic forces to inertial forces is therefore inversely proportional to the diameter, and small particles experience more aerodynamic forces per 
unit mass than large particles.

The particle volume fraction profile along the vertical centerline at $z / D=50$ is shown in figure 8a. The line is marked in figure 3 as "line". The maxima of these curves lie on the trajectories shown in figure 7. For large particle diameters, four-way coupling increases the spray penetration, and the profiles for four-way coupling are simply moved to larger $y / D$ values. For smaller particle diameters, however, four-way coupling also results in a higher peak, indicating decreased lateral dispersion. Figure $8 \mathrm{~b}$ shows transverse $v$ velocity profiles along the same line. The peak in transverse velocities can be related to the strength of the counter-rotating vortex pair (CVP) that forms in such flows [36, 48]: It is the upwash between the two vortices of the CVP. The CVP location and the turbulence levels in it are highly relevant for spray dispersion and mixing applications, and these impacts on the flow are therefore discussed here. The $y / D$ position of the peak gives the height of the vortex curve [49]. The momentum transfer from the dispersed phase to the continuous phase is slower for four-way coupling due to the lower drag on particles. This decreases the particle acceleration and thus also the source terms in the momentum equations. Hence four-way coupling decreases the peak upwash (ordinate of maxima) and increases the penetration of the vortex curve (abscissa of maxima). Additionally, the upwash zone is wider, i.e. large velocities are maintained over broader regions. These effects are stronger for the larger particles $\left(D_{p}=40 \mu \mathrm{m}\right)$.

The fluctuation levels in figures $8 \mathrm{c}$ and $8 \mathrm{~d}$ are substantially lower for the cases with four-way coupling as compared to corresponding cases with two-way coupling. A significant part of the turbulence is generated by the particles in this flow and the turbulence production is smaller if four-way coupling is accounted for.

The effect of four-way coupling on the probability density function (PDF) of the particle volume fraction for $D_{p}=40 \mu \mathrm{m}$ is given in figure 9. As the flow fields differ significantly due to the higher trajectories in the case of four-way coupling, it is not appropriate to compare PDFs for points at physically the same location. This is apparent in figure 8a: The peak particle volume fraction for four-way coupling lies at $y / D \sim 36$ whereas the profile for two-way coupling has dropped to low values. Instead, we compare the PDFs at the peak particle volume fractions on the centerline at $z / D=50$, i.e. at the maxima of each curve in figure 8a. For two-way coupling, there is high probability density for low volume fractions and smooth probability densities for larger volume fractions. For four-way coupling, the probability density of very low volume fraction is decreased, but also the probability density for volume fractions larger than 0.02. This results in a more pronounced peak in the four-way coupling scenario. This observation is consistent with the fact that turbulence levels are higher in the case of two-way coupling, and hence one expects a larger spread in the PDF and pockets of particle-free gas. 

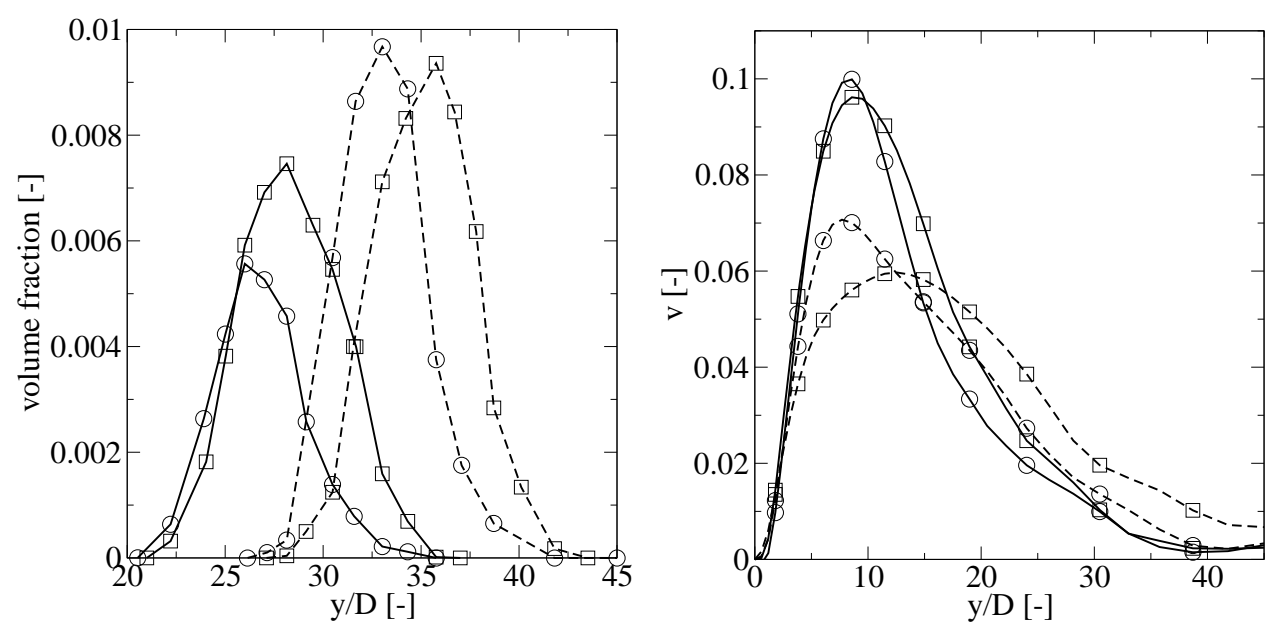

a) Volume fraction

b) Upwash
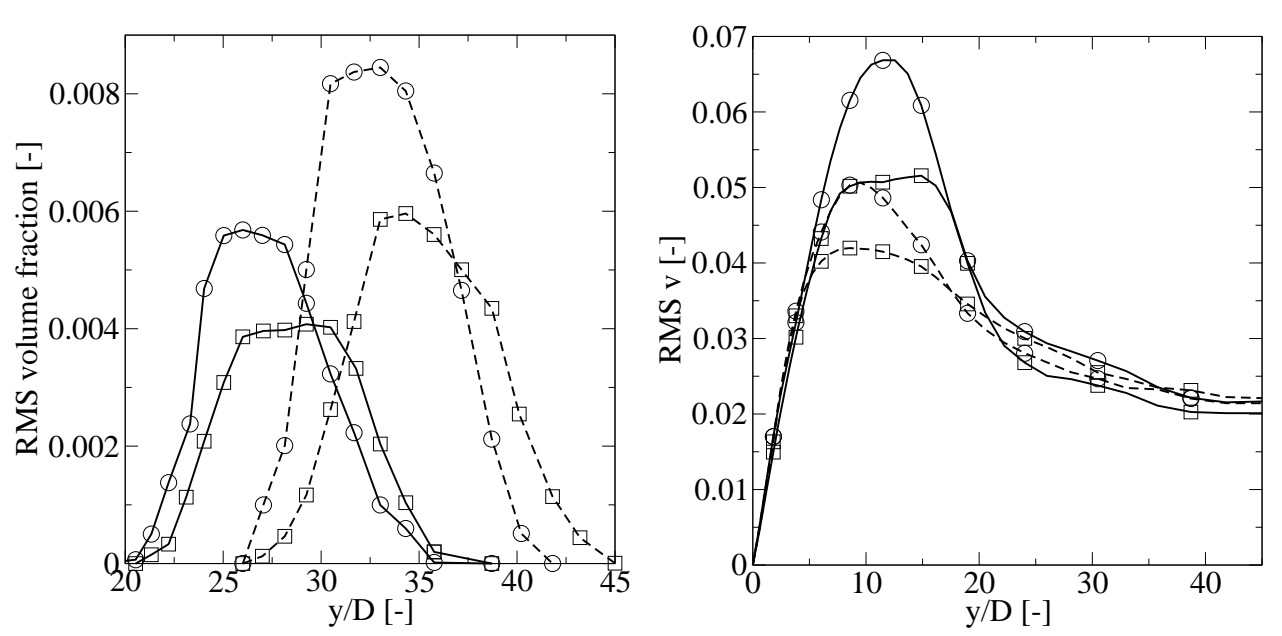

c) RMS volume fraction

d) RMS Upwash

Figure 8: Vertical centerline profiles at $\mathrm{z} / \mathrm{D}=50,-D_{p}=20 \mu m,---D_{p}=$ $40 \mu \mathrm{m}, \bigcirc$ two-way coupling, $\square$ four-way coupling 


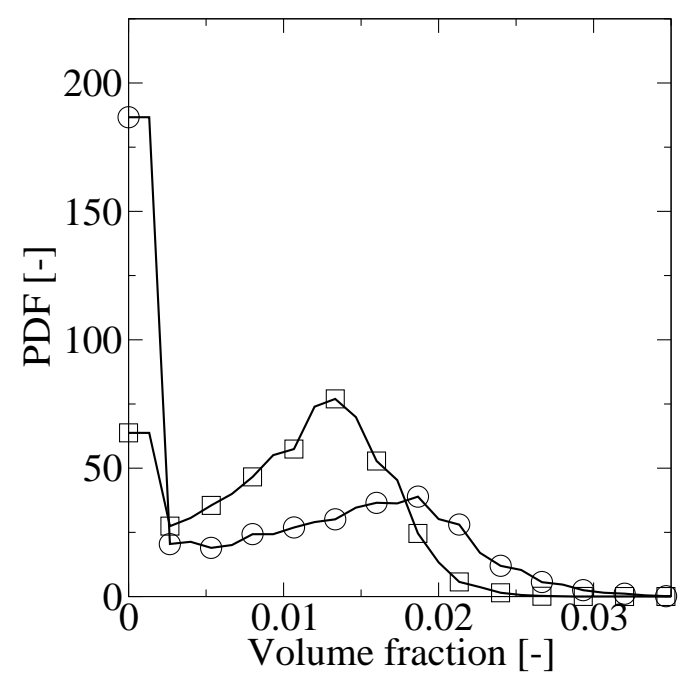

Figure 9: PDF, $D_{p}=40 \mu m \bigcirc$ two-way coupling, $\square$ four-way coupling

\subsection{Stochastic Particle Treatment}

In fuel spray applications, it is frequently desired to model atomizing sprays. In this case, stable particle diameters are on the order of a few microns. The particle number then becomes very large since the particle number is inversely proportional to the third power of the particle diameter for a given volume fraction. It is therefore computationally inefficient to track each particle and hence one defines computational parcels [29]: A computational parcel consists of a number of particles $N_{p}$ with identical properties. In simulations with a particle breakup model, $N_{p}$ can vary by many orders of magnitude. It is necessary to address the sensitivity of the four-way coupling model to the number of particles in a parcel. This number is a purely computational parameter with no physical significance.

Figure 10a displays the drag correction factors for $D_{p}=20 \mu \mathrm{m}$ with $N_{p}=10$, i.e. each computational parcel contains 10 particles (monodisperse). The drag reduction is similar, though slightly less pronounced, as with the corresponding non-stochastic treatment (figure 5a). Figure 10b is the equivalent map for the drag correction factor for the larger particles. As there are about eight times as many particles in the case with $D_{p}=20 \mu \mathrm{m}$ as compared to $D_{p}=40 \mu \mathrm{m}$, we can afford to also test the stochastic treatment such that one computational parcel represents $N_{p}=0.1$ physical particles. The simulation time is then about as long as in the case of $D_{p}=20 \mu m$ and $N_{p}=1$. As expected, the drag correction factors are about the same for non-stochastic and stochastic treatment, but the 

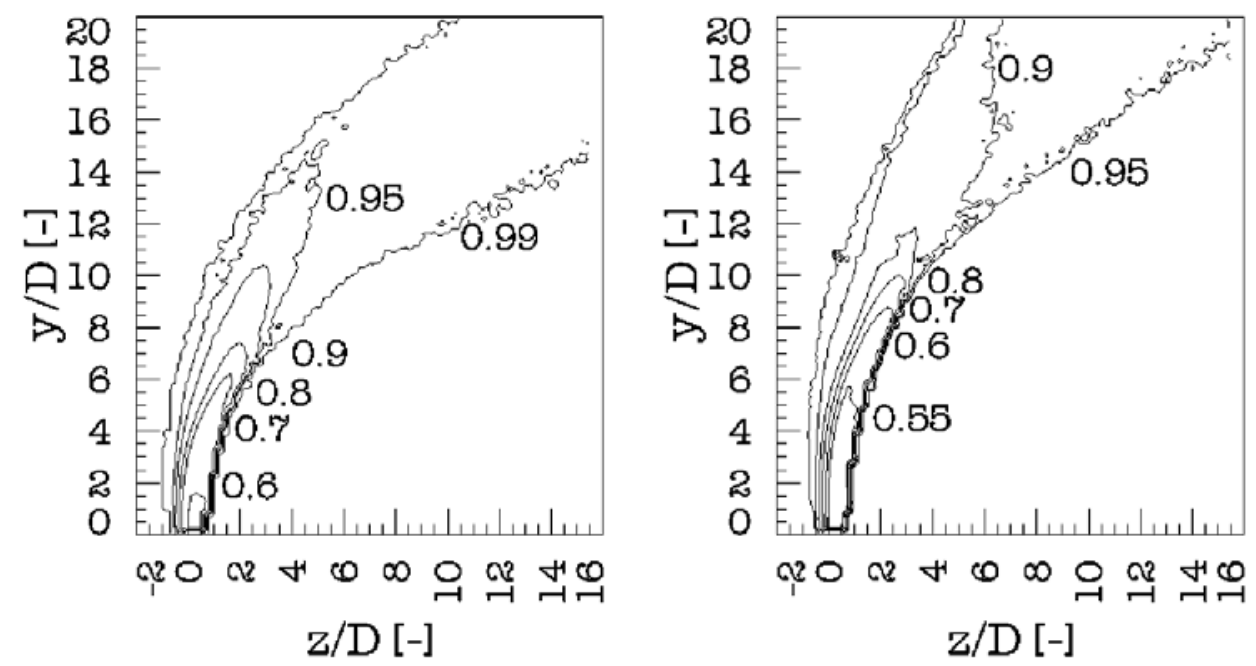
a) $D=20 \mu m, N_{p}=10$
b) $D=40 \mu m, N_{p}=0.1$

Figure 10: Drag correction factors for stochastic treatment of four-way coupling

trend in the slight difference is opposite, i.e. the isolines circumscribe generally larger areas as compared to non-stochastic treatment.

Figure 11 reveals the changes of the profiles discussed in section 5.1 due to stochastic treatment of the particles. Ideally, the curves for stochastic and nonstochastic treatment would overlap. The differences between the stochastic and non-stochastic particle treatment with four-way coupling is smaller than the difference between two-way and four-way coupling (figure 8) for the averages of volume fraction (figure 11a) and upwash velocity (figure 11b). The largest error due to stochastic modeling occurs in the fluctuations for $D=20 \mu \mathrm{m}$ (figures 11c and 11d). The fluctuations increase due to stochastic sampling (which is a computational parameter) whereas four-way coupling (a modeling parameter) decreases the fluctuation. This is an example of two effects which partly cancel and therefore mask each other.

\subsection{Four-Way Coupling for a Polydisperse Spray}

If the particles are allowed to break up, the spray can obviously not be monodisperse. We model droplet breakup taking into account stripping breakup and bag breakup [30]. However, the drag correction factors have been computed for a pair of spheres with equal diameter. Additional modeling is therefore required to account for the droplet size variation in polydisperse spray. Chen and $\mathrm{Wu}[20]$ computed the flow around two spheres with a diameter ratio of $1 / 2$, but drag 

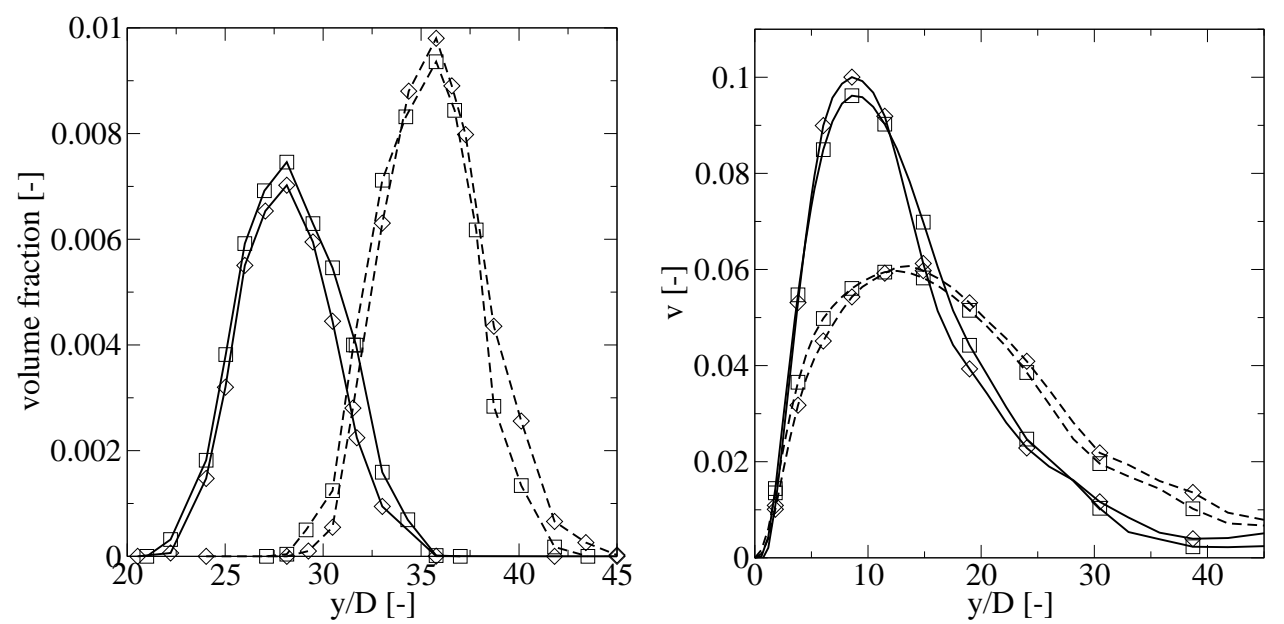

a) Volume fraction

b) Upwash velocity
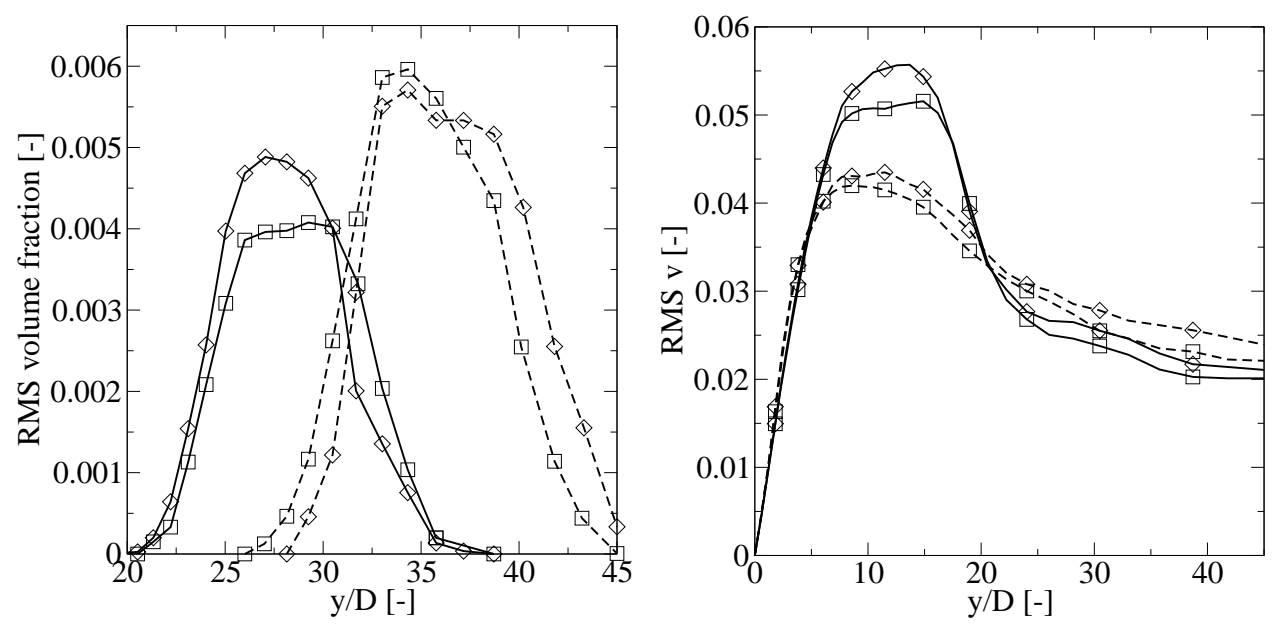

c) RMS volume fraction

d) RMS Upwash velocity

Figure 11: Vertical centerline profiles at $\mathrm{z} / \mathrm{D}=50,-D_{p}=20 \mu m,---D_{p}=$ $40 \mu m, \diamond$ stochastic, $\square$ non-stochastic 


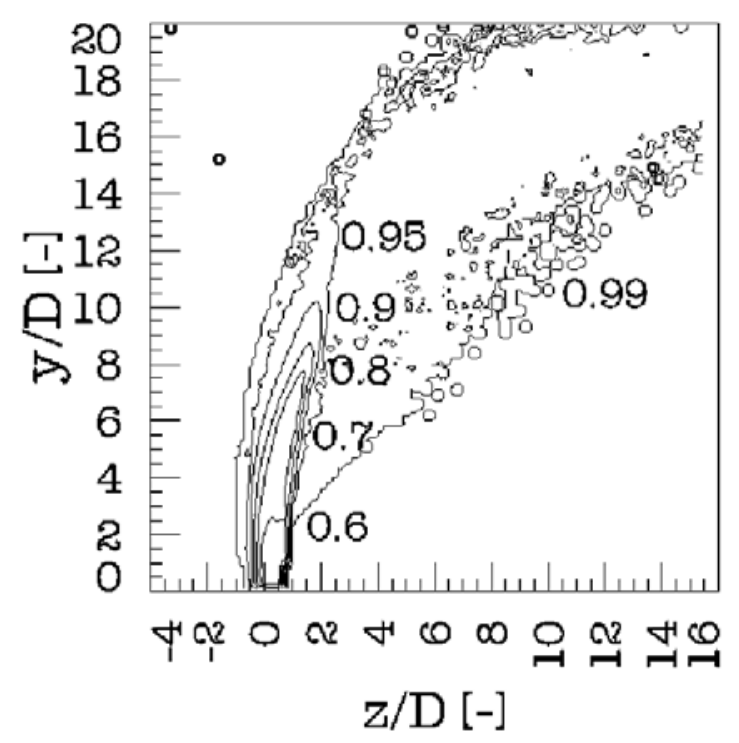

Figure 12: Drag correction factors for modeling of droplet breakup

correction factors for other droplet size ratios are not available. The look-up tables would also be cumbersome due to the extra dimension. Our model allowing for polydisperse spray is therefore ad hoc. We model the interaction of two droplets of different diameters $D_{p, 1}$ and $D_{p, 2}$ by finding their geometric mean diameter $D_{p, g e o}$ :

$$
D_{p, g e o}=\sqrt{D_{p, 1} D_{p, 2}}
$$

This geometric mean diameter is used as representative diameter of the droplet pair in the four-way coupling model. If two droplets with identical diameters interact, the geometric mean diameter is simply the diameter of the droplets, and thus the extra model for varying droplet size vanishes for this case. The more the diameter of the two interacting droplets varies, the larger the modeling error of the extra model accounting for polydisperse spray becomes.

In figure 12, the drag correction factors for the polydisperse spray with breakup are plotted. The injection diameter is $D_{p}=40 \mu \mathrm{m}$, implying an injection Weber number on the order of $W e \sim 100$. The Weber number is defined in equation (22) where $\sigma$ is the surface tension.

$$
W e=\frac{\rho_{c} D_{p}|\vec{v}-\vec{u}|^{2}}{\sigma}
$$

Droplets therefore break up rapidly under the given conditions. The effect of four-way coupling on the particle drag is slightly decreased if breakup is included 
(the drag reductions are smaller), as the areas within corresponding isolines are smaller.

The velocity and volume fraction profiles at $z / D=50$ are compared for particles (which do not break up) and droplets in figure 13. The injection diameter in both cases is $D_{p}=40 \mu m$. Droplet breakup has strong effects on the flow as is well-known: The peak in droplet volume fraction (figure 13a) is reduced by an order of magnitude due to enhanced lateral dispersion of smaller droplets. The induced upwash velocities (figure 13b) are larger due to the smaller Stokes numbers in the case of small droplets.

The effect of four-way coupling is reversed if droplet breakup is included. For particles, four-way coupling decreases the upwash velocities (figure $8 \mathrm{~b}$ ) and the fluctuations in volume fraction (figure 8c) and upwash velocities (figure 8d) as discussed in section 5.1. Contrarily, if droplet breakup is included, four-way coupling results in increased upwash velocities (figure 13b) and increased fluctuations in volume fraction (figure 13c) and upwash velocities (figure 13d). The reason is the increased slip velocities in the case of four-way coupling which lead to larger Weber numbers and hence to more rapid droplet breakup and smaller particle sizes. The faster breakup results in increased momentum transfer rates from the droplets to the continuous phase, resulting in the larger upwash velocities and their fluctuations. Therefore, also the fluctuation in volume fraction is larger.

\section{Conclusions}

The effects of a four-way coupling model for describing aerodynamic particle interaction have been discussed. To elaborate on the impact of the model more clearly, an idealized situation has been considered initially: The particles are supposed to be monodisperse, rigid spheres. In particular, direct application to spray would require polydisperse particles which are allowed to break up, collide, and evaporate. As our microscopic model for aerodynamic particle interaction is based on flow around a pair of particles, we only allow one interaction per particle and disregard many-body interaction. A domain decomposition technique reduces the computational cost introducing only a small error. Under such conditions, which will give a lower estimate for the impact of aerodynamic particle interaction, we find that the drag on particles decreases by more than $40 \%$ in the near-field of a particle jet in crossflow due to aerodynamic four-way coupling. As far as 15 nozzle diameters from the nozzle, the average drag on particles is still reduced by $10 \%$. The penetration of monodisperse particles into the crossflow is therefore larger if four-way coupling is employed. The induced upwash of the CVP is smaller due to the decreased drag on the droplets. The fluctuations are smaller in the case of four-way coupling as not as much turbulence is generated. Since we seek to eventually apply our model to more realistic sprays, we undertake initial steps in this direction. In particular, sprays are never monodisperse and contain such a large number of droplets that stochastic methods usually have to be applied. Stochastic modeling of the particles is shown to have rela- 

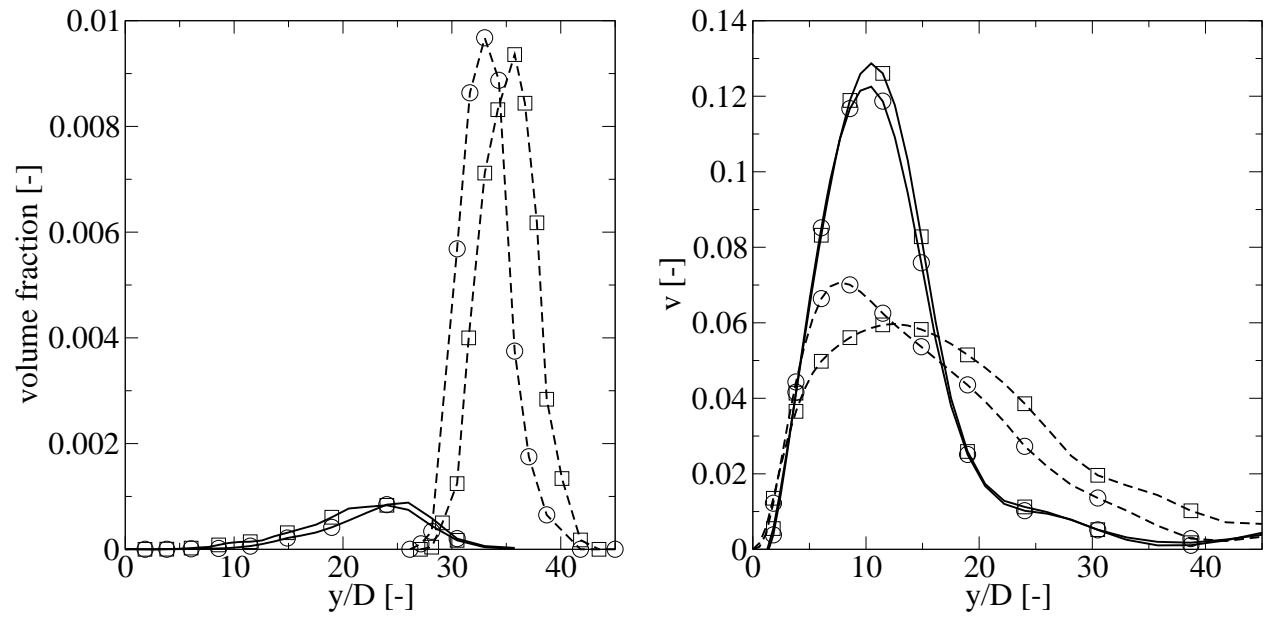

a) Volume fraction

b) Upwash velocity
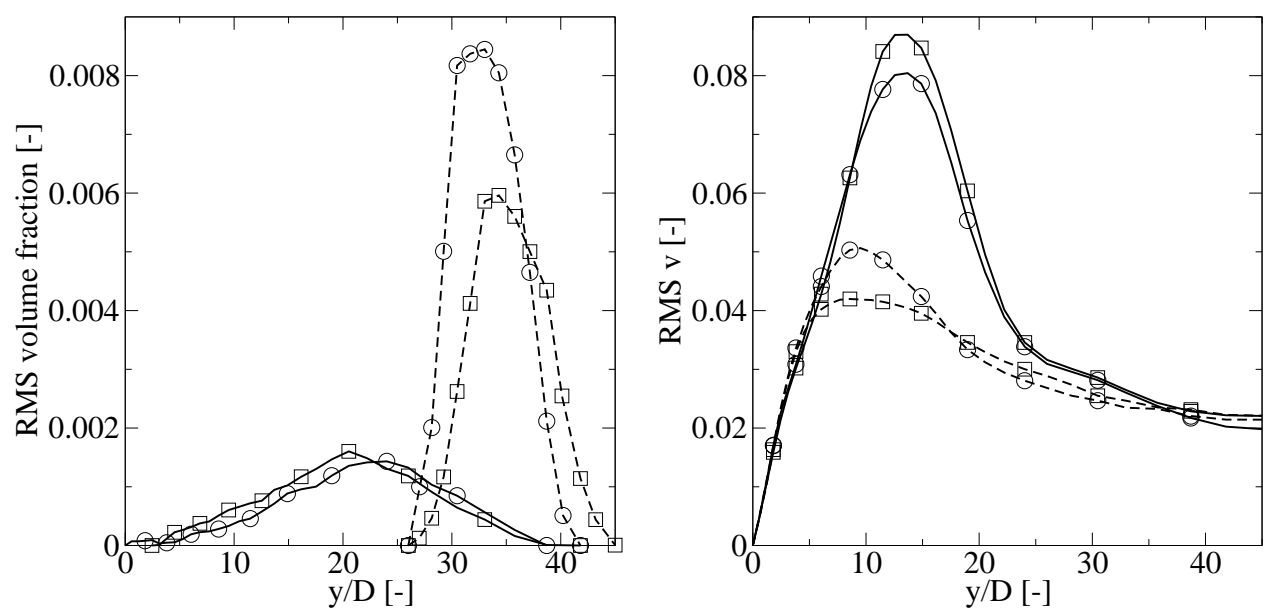

c) RMS volume fraction

d) RMS Upwash velocity

Figure 13: Transverse centerline profiles at $\mathrm{z} / \mathrm{D}=50,-$ droplets, --- particles, $\circ$ two-way coupling, $\square$ four-way coupling 
tively small effects on the four-way coupling model over two order of magnitude covering 0.1 to 10 particles in a parcel. Finally, we study a polydisperse spray by including a breakup model. We suggest an ad hoc model to handle polydisperse sprays in the four-way coupling model. Under such conditions, four-way coupling increases the induced upwash and the fluctuations of the upwash velocity: The effect of four-way coupling is reversed in the case with breakup since the four-way coupling leads to larger Weber numbers and hence more rapid droplet breakup and smaller particle sizes.

\section{Acknowledgements}

The project work was partially supported by the Swedish Energy Agency (STEM) and partially by the Centre for Combustion Science and Technology (CECOST) through the SSF program. Computational recourses were provided by LUNARC computing center at Lund University and HPC2N facilities within the SNAC/SNIC allocation program. 


\section{Appendix}

\begin{tabular}{|c|c|c|c|c||c|c|c|c|c|}
\hline Angle & $L / D=1.5$ & $L / D=3$ & $L / D=4.5$ & $L / D=6$ & Angle & $L / D=1.5$ & $L / D=3$ & $L / D=4.5$ & $L / D=6$ \\
\hline 0 & 0.421 & 0.568 & 0.648 & 0.702 & 0 & 0.343 & 0.513 & 0.593 & 0.649 \\
\hline 15 & 0.554 & 0.825 & 0.933 & 0.976 & 15 & 0.595 & 0.880 & 0.967 & 0.992 \\
\hline 30 & 0.755 & 0.976 & 0.999 & 1.002 & 30 & 0.819 & 0.984 & 0.999 & 1.001 \\
\hline 45 & 0.870 & 0.997 & 1.002 & 1.002 & 45 & 0.890 & 0.995 & 1.001 & 1.001 \\
\hline 60 & 0.948 & 1.004 & 1.004 & 1.002 & 60 & 0.952 & 1.003 & 1.003 & 1.002 \\
\hline 75 & 1.009 & 1.010 & 1.005 & 1.003 & 75 & 1.014 & 1.009 & 1.005 & 1.003 \\
\hline 90 & 1.057 & 1.013 & 1.005 & 1.003 & 90 & 1.068 & 1.014 & 1.005 & 1.003 \\
\hline 105 & 1.074 & 1.012 & 1.005 & 1.002 & 105 & 1.092 & 1.014 & 1.005 & 1.002 \\
\hline 120 & 1.054 & 1.006 & 1.002 & 1.001 & 120 & 1.080 & 1.009 & 1.003 & 1.001 \\
\hline 135 & 1.007 & 0.995 & 0.998 & 0.999 & 135 & 1.041 & 0.999 & 0.999 & 0.999 \\
\hline 150 & 0.938 & 0.980 & 0.993 & 0.997 & 150 & 0.973 & 0.983 & 0.993 & 1.001 \\
\hline 165 & 0.906 & 0.963 & 0.987 & 0.994 & 165 & 0.919 & 0.960 & 0.986 & 0.994 \\
\hline 180 & 0.904 & 0.958 & 0.985 & 0.994 & 180 & 0.918 & 0.949 & 0.982 & 0.993 \\
\hline
\end{tabular}

$\begin{array}{ll}\text { a) } R e_{p}=50 & \text { b) } R e_{p}=100\end{array}$

\begin{tabular}{|c|c|c|c|c|}
\hline Angle & $L / D=1.5$ & $L / D=3$ & $L / D=4.5$ & $L / D=6$ \\
\hline 0 & 0.2102 & 0.4468 & 0.5377 & 0.5879 \\
\hline 15 & 0.6686 & 0.9479 & 0.9927 & 0.9990 \\
\hline 30 & 0.8738 & 0.9864 & 0.9986 & 1.0028 \\
\hline 45 & 0.8993 & 0.9882 & 0.9996 & 1.0002 \\
\hline 60 & 0.9632 & 1.0040 & 1.0035 & 1.0022 \\
\hline 75 & 1.0303 & 1.0146 & 1.0056 & 1.0032 \\
\hline 90 & 1.0910 & 1.0185 & 1.0065 & 1.0038 \\
\hline 105 & 1.1139 & 1.0189 & 1.0070 & 1.0031 \\
\hline 120 & 1.1047 & 1.0179 & 1.0052 & 1.0022 \\
\hline 135 & 1.0897 & 1.0117 & 1.0015 & 1.0000 \\
\hline 150 & 1.0450 & 0.9958 & 0.9942 & 0.9968 \\
\hline 165 & 0.9768 & 0.9618 & 0.9824 & 0.9925 \\
\hline 180 & 0.9668 & 0.9218 & 0.9705 & 0.9900 \\
\hline
\end{tabular}

c) $R e_{p}=200$

Figure 14: Drag correction factors $f$ for $R e_{p}=50,100,200$ [22] 


\section{References}

[1] Wu P K, Kirkendall K, Fuller R and Nejad A 1997 J. Propulsion and Power 13 64-73

[2] Wu P, Kirkendall K, Fuller R and Nejad A 1998 J. Propulsion and Power $14173-182$

[3] Ghosh S and Hunt J 1998 J. Fluid Mech. 365 109-136

[4] Caraeni D, Bergström C and Fuchs L 2000 Flow, Turbulence and Combustion $65223-244$

[5] Madabhushi R 2003 Atomization and Sprays 13 413-424

[6] Lefebvre A 1995 J. Eng. for Gas Turbines and Power 117 617-654

[7] Hirt C and Nichols B 1981 J. Comp. Phys. 39 201-225

[8] Gueyffier D, Li J, Nadim A, Scardovelli R and Zaleski S 1999 J. Comp. Phys. 152 423-456

[9] Crowe C, Sommerfeld M and Tsuji Y 1998 Multiphase Flows with Droplets and Particles (CRC Press)

[10] Sirignano W 1999 Fluid Dynamics and Transport of Droplets and Sprays (Cambridge University Press)

[11] Elghobashi S 1994 Appl. Sci. Res. 52 309-329

[12] Salewski M and Fuchs L 2007 Int. J. Multiphase Flow 33 394-410

[13] Tsuji Y, Morikawa Y and Terashima K 1982 Int. J. Multiphase Flow 8 $71-82$

[14] Chiang C and Sirignano W 1993 Int. J. Heat Mass Transfer. 36 875-886

[15] Kim I, Elghobashi S and Sirignano W 1993 J. Fluid Mech. 246 465-488

[16] Zhu C, Liang S and Fan L 1994 Int. J. Multiphase Flow 20 117-129

[17] Liang S, Hong T and Fan L 1996 Int. J. Multiphase Flow 22 285-306

[18] Olsson M and Fuchs L 1998 The Interaction of Spherical Particles in a Fluid Flow Governed by the Navier-Stokes Equations Proc. of the 4th ECCOMAS Conference on Computational Fluid Dynamics, Papailion et al. (eds.) (John Wiley and Sons)

[19] Folkersma R, Stein H and van de Vosse F 2000 Int. J. Multiphase Flow 26 $877-887$

[20] Chen R and Wu J 2000 Chem.Eng.Sci. 55 1143-1158 
[21] Tsuji T, Narutomi R, Yokomine T, Ebara S and Shimizu A 2003 Int. J. Multiphase Flow 29 1431-1450

[22] Prahl L, Hölzer A, Arlov D, Revstedt J, Sommerfeld M and Fuchs L 2007 Int. J. Multiphase Flow 33 707-725

[23] Holländer W and Zaripov S 2005 Int. J. Multiphase Flow 31 53-68

[24] Chen R and Lu Y 1999 Int. J. Multiphase Flow 25 1645-1655

[25] Silverman I and Sirignano W 1994 Int. J. Multiphase Flow 20 99-116

[26] Wang L P, Ayala O, Kasprzak S and Grabowski W 2005 J. Atmos. Sci. 62 $2433-2450$

[27] Wang L P, Ayala O and Grabowski W 20078 1-28

[28] Ayala O, Grabowski W W and Wang L P 2007 J. Comp. Phys. 225 51-73

[29] Dukowicz J 1980 J. Comp. Phys. 35 229-253

[30] Reitz R and Diwakar R 1986 SAE Technical Paper 860469

[31] Maxey M and Riley J 1983 Phys. Fluids 26 883-889

[32] Sommerfeld M 2001 Int. J. Multiphase Flow 27 1829-1858

[33] Fureby C and Grinstein F 2002 J. Comp. Phys. 181 68-97

[34] Olsson M and Fuchs L 1998 Phys. Fluids 10 476-486

[35] Gullbrand J, Bai X S and Fuchs L 2001 Int. J. Numerical Methods in Fluids 36 687-709

[36] Salewski M, Stankovic D and Fuchs L 2008 Flow, Turbulence and Combustion $80255-283$

[37] Jiang G S and Shu C W 1996 J. Comp. Phys. 126 202-228

[38] Kravchenko A and Moin P 1997 J. Comp. Phys. 131 310-322

[39] Ghosal S 1996 J. Comp. Phys. 125 187-206

[40] Wang Q and Squires K 1996 Int. J. Multiphase Flow 22 667-683

[41] Yeh F and Lei U 1981 Phys. Fluids 3 2571-2586

[42] Becker J and Hassa C 2002 Atomization and Sprays 11 49-67

[43] Rachner M, Becker J, Hassa C and Doerr T 2002 Aerospace Science and Technology 6 495-506 
[44] Khosla S and Crocker D 2004 CFD Modeling of the Atomization of plain Liquid Jets in Cross Flow for Gas Turbine Applications, GT2004-54269 ASME Turbo Expo 2004 Proceedings

[45] Salewski M and Fuchs L 2006 Dispersion of Circular, Non-circular, and Swirling Spray Jets in Crossflow Direct and Large-Eddy Simulation VI (ERCOFTAC Series), Lamballais, E., Friedrich, R., Geurts,B.J., Metais, O. (Eds.) (dordrecht, The Netherlands: Springer) ISBN 978-1-4020-4909-5

[46] Salewski M, Stankovic D and Fuchs L 2007 J. Eng. Gas Turbines and Power 129

[47] Salewski M, Revstedt J and Fuchs L 2007 Droplet Deformation Effects in Lagrangian Particle Tracking of a Spray Jet in Crossflow In Proceedings of the 6th International Conference on Multiphase Flow

[48] Fric T and Roshko A 1994 J. Fluid Mech. 279 1-47

[49] Fearn R and Weston R 1974 AIAA J. 12 1666-1671 